\title{
Clinical Study \\ Local and Systemic Inflammatory Responses to Experimentally Induced Gingivitis
}

\author{
Shaneen J. Leishman, ${ }^{1}$ Gregory J. Seymour, ${ }^{2,3}$ and Pauline J. Ford ${ }^{1}$ \\ ${ }^{1}$ The University of Queensland, School of Dentistry, Brisbane QLD 4072, Australia \\ ${ }^{2}$ The University of Queensland, School of Medicine, Herston QLD 4006, Australia \\ ${ }^{3}$ Sir John Walsh Research Institute, University of Otago, Dunedin 9054, New Zealand
}

Correspondence should be addressed to Shaneen J. Leishman; s.leishman@uq.edu.au

Received 14 June 2013; Revised 10 September 2013; Accepted 30 September 2013

Academic Editor: Fabrizia Bamonti

Copyright (C) 2013 Shaneen J. Leishman et al. This is an open access article distributed under the Creative Commons Attribution License, which permits unrestricted use, distribution, and reproduction in any medium, provided the original work is properly cited.

\begin{abstract}
This study profiled the local and systemic inflammatory responses to experimentally induced gingivitis. Eight females participated in a 21-day experimental gingivitis model followed by a 14-day resolution phase. Bleeding on probing and plaque index scores were assessed before, during, and after resolution of gingival inflammation, and samples of saliva, GCF, and plasma were collected. Samples were assessed for biomarkers of inflammation using the BioPlex platform and ELISA. There were no significant changes in GCF levels of cytokines during the experimental phase; however, individual variability in cytokine profiles was noted. During resolution, mean GCF levels of IL-2, IL-6, and TNF- $\alpha$ decreased and were significantly lower than baseline levels $(P=0.003$, $P=0.025$, and $P=0.007$, resp.). Furthermore, changes in GCF levels of IL-2, IL-6, and TNF- $\alpha$ during resolution correlated with changes in plaque index scores $(r=0.88, P=0.004 ; r=0.72, P=0.042 ; r=0.79, P=0.019$, resp.). Plasma levels of sICAM-1 increased significantly during the experimental phase $(P=0.002)$ and remained elevated and significantly higher than baseline levels during resolution $(P<0.001)$. These results support the concept that gingivitis adds to the systemic inflammatory burden of an individual.
\end{abstract}

\section{Introduction}

There is currently widespread interest in the relationship between periodontal disease and cardiovascular disease (CVD); however, despite this, the relationship remains to be established as a causal one, and longitudinal and mechanistic studies are required to strengthen the evidence available.

The anatomical proximity of the bacterial biofilm to the periodontal vasculature facilitates the systemic dissemination of bacteria and host products [1]. Thus, it is proposed that local inflammation within the oral cavity, paralleled by transient bacteraemia, adds to the systemic inflammatory burden of an individual, promoting systemic disease. In the case of CVD, circulating inflammatory mediators may activate the vascular endothelium, enhancing the development of atherosclerosis (reviewed in Seymour et al. [2]). Indeed, cardiovascular (CV) risk has been associated with increased levels of interleukin-1 (IL-1), IL-6, tumour necrosis factoralpha (TNF- $\alpha$ ), soluble intercellular adhesion molecule-1
(sICAM-1), and acute-phase proteins such as C-reactive protein (CRP) and fibrinogen [3-6]. Furthermore, plasma levels of inflammatory markers have been shown to be stronger predictors of future $\mathrm{CV}$ events than low density lipoprotein (LDL) levels [7].

Periodontitis patients have increased systemic levels of inflammatory markers (IL-6, CRP, haptoglobin, and leukocytosis) [8-11] and exhibit dyslipidemia and endothelial dysfunction [11, 12], all of which can be reduced by periodontal therapy $[13,14]$. Complete resolution of periodontitis by extraction of all teeth has also been shown to result in significant reductions in a number of $\mathrm{CV}$ risk factors such as CRP, plasminogen activator inhibitor-1 (PAI-1), and fibrinogen, particularly in nonsmokers [15].

While a number of studies have investigated the systemic effects of periodontitis, relatively less is known regarding the systemic effects of gingivitis. Gingivitis is inflammation of the gingival tissues only and, unlike periodontitis, does not involve attachment or alveolar bone loss. Clinical signs 
include gingival redness, swelling, and bleeding upon probing of the gingival sulcus during clinical examination. It is initiated by the local accumulation of bacteria (dental plaque) on periodontal surfaces [16], but the host inflammatory response to the bacterial challenge is an important determinant of disease severity (reviewed in Gemmell et al. [17]). Gingivitis is ubiquitous and has the potential to contribute significantly to the systemic inflammatory burden of an individual. As gingivitis is rapidly inducible and reversible [16] it is an appropriate model to assess the longitudinal effects of oral inflammation on systemic inflammation.

The gingival crevicular fluid (GCF) is an inflammatory exudate produced as a result of inflammation within the gingival tissues and flows continually into the gingival sulcus. This flow is increased during gingivitis and periodontitis. The GCF is enriched with bacterial and host products and, therefore, reflects the inflammatory changes occurring within the gingival tissues. Within the oral cavity, GCF mixes with secretions from the salivary glands and adds to the whole saliva. Gingivitis is associated with increased GCF levels of inflammatory mediators including IL-1 $\beta$ [18-22]; however, despite this, serum changes in cytokines are yet to be reported $[19,23,24]$. However, studies have shown endotoxemia and an increased peripheral leukocyte response to the accumulation of dental plaque $[23,25,26]$. The aim of the present study, therefore, was to use the full-mouth experimental gingivitis model to investigate whether the accumulation of dental plaque led to a systemic inflammatory response that could be detected within oral fluids.

\section{Materials and Methods}

2.1. Subjects. Eight nonsmoking, systemically healthy female students of the University of Queensland (25.5 \pm 7.1 years; range 17-41 years), with no history of periodontal disease, participated in the study. Exclusion criteria included subjects requiring antibiotic cover for dental treatment, those who had antibiotics in the preceding 3 months, or those who were pregnant. Institutional ethics committee approval was obtained to carry out the study. A written explanation of the purpose of the study was provided, and signed informed consent, according to the Helsinki Declaration, was obtained from each subject prior to entry into the study.

2.2. Clinical Study Design. Gingival inflammation was induced in subjects using the experimental gingivitis model developed by Löe et al. [16] (Figure 1(a)). At baseline (day 0 ), subjects underwent a clinical assessment at which time bleeding on probing (BOP) across the full mouth was used to assess gingival inflammation. BOP was examined at six sites per tooth and expressed as a percentage of the total number of sites examined. The plaque index of Silness and Löe [27] was used to assess full-mouth plaque accumulation. Subsequently, subjects were asked to abstain from all oral hygiene procedures, including chewing gum, for a period of 21 days. At day 21, subjects underwent a second clinical assessment, received a professional prophylaxis, and were instructed to resume normal hygiene practices. Subjects also received an Oral-B Triumph powered toothbrush (Procter \& Gamble, Cincinnati, OH, USA) to facilitate the resolution of gingival inflammation. Fourteen days later (day 35), subjects underwent a final clinical assessment to confirm the restoration of gingival health. To standardise the sample collection procedure, all participants were requested to provide a diet summary for the $24 \mathrm{~h}$ period prior to the appointment. All subjects were given morning appointments and asked not to eat or drink anything except water after 9 p.m. on the previous day. Finally, subjects were asked to floss and brush their teeth $2 \mathrm{~h}$ prior to each appointment, except prior to attending on day 21 .

2.3. Collection of GCF, Saliva, and Plasma Samples. At each visit, samples of peripheral venous blood, saliva, and GCF were collected. GCF was collected from sites that reflected the gingival status, either the upper premolars or lower incisors. A Millipore filter paper strip with $1 \mathrm{~mm}$ width was inserted into the gingival sulcus (Figure 1(b)), left for $30 \mathrm{~s}$ (or replaced when almost saturated up to a total sample time of $30 \mathrm{~s}$ ) and placed in a sterile tube containing $180 \mu \mathrm{L}$ of buffer $\left(10 \mathrm{mM} \mathrm{NaH}_{2} \mathrm{PO}_{4}\right.$ and $150 \mathrm{mM} \mathrm{NaCl}$, at $\mathrm{pH}$ 7.2). Strips visibly contaminated by bleeding were discarded and another sample collected. Unstimulated saliva $(2-4 \mathrm{~mL})$ was collected from each individual by allowing saliva to pool in the floor of the mouth followed by expectoration into sterile specimen tubes and then was aliquoted. GCF and saliva samples were stored at $-20^{\circ} \mathrm{C}$ before being processed.

2.4. Preparation of GCF, Saliva, and Plasma Samples. GCF and saliva samples were centrifuged ( $800 \mathrm{~g}$ for $10 \mathrm{~min}$ ) and the supernatants were retained and stored at $-80^{\circ} \mathrm{C}$. Plasma was isolated from peripheral blood following Ficoll-Paque (Pharmacia LKB, Uppsala, Sweden) gradient centrifugation.

2.5. Assessment of Inflammatory Mediators. GCF, saliva, and plasma samples were assessed for levels of cytokines (IL- $1 \beta$, IL-2, IL-4, IL-5, IL-6, IL-10, IL-12(p70), IL-13, IFN- $\gamma$, and TNF- $\alpha$ ) using a BioPlex cytokine assay kit in combination with the BioPlex manager software (Bio-Rad, Hercules, CA, USA) as well as for CRP and sICAM-1 using high-sensitivity enzyme linked immunosorbent assay (ELISA) kits (Bender MedSystems, Burlingame, CA, USA) according to the manufacturers' instructions. Results are reported as standard curve units (cytokines: pg/mL; CRP and sICAM-1: ng/mL) [22].

2.6. Statistical Analysis. It has been reported that methods based on the $t$-distribution are more suitable for the analysis of continuous data from small samples than rank (nonparametric) methods [28]. As such, repeated measures general linear models with Bonferroni adjustment for multiple comparisons were used to test for differences in clinical indices and mediator levels over time. Specific changes were examined between baseline and peak of induction (day 21), between days 21 and 35 (resolution of disease), and between day 35 and baseline. For two subjects, the analysis of saliva using the BioPlex system was not possible due to technical reasons. Therefore, the statistical analysis of 


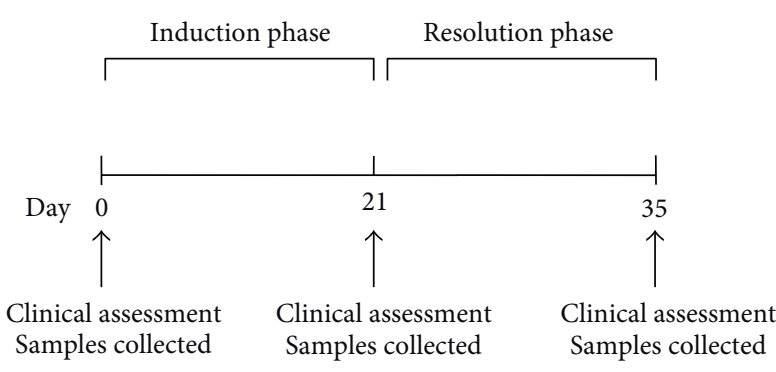

(a)

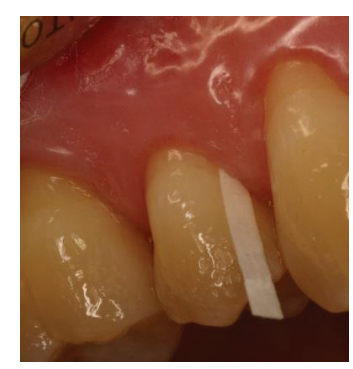

(b)

Figure 1: (a) Study design and (b) GCF collection.

saliva, with regard to cytokine levels, was performed on six subjects. Pearson correlations were used to examine the associations between clinical indices and mediator levels. Statistical analyses were carried out using SPSS v19.0 (SPSS Inc, Chicago, IL, USA).

\section{Results}

3.1. Clinical Assessment. The mean BOP and plaque index scores for each visit are shown in Table 1 . At baseline, six of the eight subjects had naturally occurring gingivitis ( $>10 \% \mathrm{BOP})$.

The results of the general linear model indicated that there was a significant change in BOP over time $(P=0.001)$ (Table 1). Gingival inflammation was significantly induced at day 21 with a 3.1-fold increase in BOP $(P=0.004)$ compared with baseline (Table 1). Following prophylaxis and resumption of oral hygiene procedures at day 21 , plaque index scores decreased 7.2-fold ( $P<0.001$; student's paired $t$-test) and BOP decreased 4.9 -fold $(P=0.003)$ at day 35 to levels lower than baseline $(P=0.005)$ (Table 1$)$.

3.2. GCF Mediator Levels during Experimental Gingivitis. Five of the twelve mediators in the GCF demonstrated changes in levels over time: IL-2 $(P=0.037)$, IL-6 $(P=$ $0.012)$, and TNF- $\alpha(P=0.03)$ at the $5 \%$ significance level and IL-1 $\beta(P=0.070)$ and IFN- $\gamma(P=0.063)$ at the $10 \%$ significance level (Table 2 ). There were no significant changes in the GCF levels of cytokines during the induction phase; however, not surprisingly, individual variability in cytokine profiles was noted during this time.

At day 35, following the resolution of gingivitis, mean levels of IL-2, IL-6, and TNF- $\alpha$ decreased 20.0-fold ( $P=$ $0.062 ; 10 \%$ significance level $), 16.0$-fold $(P=0.054 ; 10 \%$ significance level), and 16.1-fold ( $P=0.064 ; 10 \%$ significance level), respectively, in concordance with improvement in clinical indices and were significantly lower than baseline levels $(P=0.003, P=0.025$, and $P=0.007$, resp.) (Table 2). Furthermore, changes in the GCF levels of IL-2, IL-6, and TNF- $\alpha$ during the resolution phase correlated with changes in plaque index scores $(r=0.88, P=0.004 ; r=0.72$, $P=0.042 ; r=0.79, P=0.019$, resp.). Mean levels of IL$1 \beta$ and IFN $-\gamma$ at day 35 were also significantly lower than baseline levels $(P=0.004$ and $P=0.014$, resp.) (Table 2$)$.
There were no significant changes in the GCF levels of IL4, IL-5, IL-10, IL-12(p70), or IL-13 over time. GCF levels of sICAM-1 and CRP were low or undetectable (Table 2).

3.3. Salivary Mediator Levels during Experimental Gingivitis. Saliva showed higher levels of cytokines than found in GCF; however, these levels did not change over time. As found in GCF, salivary levels of sICAM-1 were low or undetectable (Table 3).

3.4. Plasma Mediator Levels during Experimental Gingivitis. sICAM-1 was the only mediator in the plasma that demonstrated significant changes in levels over time $(P<0.001)$ (Table 4). At day 21, following the cessation of oral hygiene procedures, mean levels of sICAM-1 increased 2.6-fold ( $P=$ 0.002 ), in concordance with increases in clinical indices and remained elevated and significantly higher than baseline levels during the resolution phase $(P<0.001)$ (Table 4$)$.

All subjects had detectable plasma levels of CRP at baseline. During induction, four of the eight subjects had increased levels of CRP, and all of these subjects had reduced levels at day 35 , following the resolution of gingivitis. Plasma levels of cytokines, with the exception of IL-5, were low or undetectable (Table 4).

\section{Discussion}

In the literature there is strong evidence for an association between periodontitis and systemic inflammation, but relatively less is known regarding the systemic effects of gingivitis. This study is one of only two reported studies [25] to employ the full-mouth experimental gingivitis model to investigate the systemic effects of dental plaque. Furthermore, it is the only full-mouth experimental gingivitis model to have simultaneously investigated the inflammatory responses within the GCF and saliva. One limitation of previous experimental gingivitis models has been the use of the split mouth design $[19,24]$. This design is suitable for the investigation of local responses to the accumulation of dental plaque but is not suitable when investigating systemic effects. A previous study has shown an association between serum sICAM-1 levels and clinical signs of BOP in periodontitis patients. 
TABLE 1: Clinical indices at baseline, peak of induction (day 21), and at resolution (day 35) of experimental gingivitis.

\begin{tabular}{lccccc}
\hline & Baseline & Day 21 & Day 35 & $F$ statistic & $P$ value \\
\hline Plaque Index & - & $2.14(0.12)$ & $0.30(0.07)$ & $T(7)=17.76^{*}$ & $<0.001$ \\
BOP (\%) & $12.01(1.28)$ & $37.19(5.90)$ & $7.64(1.20)$ & $(1.03,7.21)=28.79$ & $0.001^{\dagger}$ \\
\hline
\end{tabular}

Clinical indices are expressed as mean (standard error, SE).

${ }^{*} t$-statistic: Student's $t$-test.

${ }^{\dagger}$ Greenhouse-Geisser correction for nonsphericity.

TABLE 2: GCF mediator levels at baseline, peak of induction (day 21), and at resolution (day 35) of experimental gingivitis.

\begin{tabular}{|c|c|c|c|c|c|}
\hline & Baseline & Day 21 & Day 35 & $F$ statistic & $P$ value \\
\hline $\mathrm{IL}-1 \beta(\mathrm{pg} / \mathrm{mL})$ & $52.91(9.13)$ & $91.93(33.15)$ & $9.64(6.01)$ & $(1.09,7.63)=4.37$ & $0.070^{*}$ \\
\hline IL-2 (pg/mL) & $7.81(1.39)$ & $11.82(3.98)$ & $0.59(0.39)$ & $(1.16,8.11)=5.97$ & $0.037^{*}$ \\
\hline $\mathrm{IL}-4(\mathrm{pg} / \mathrm{mL})$ & $0.19(0.07)$ & $0.48(0.22)$ & $0(0)$ & $(1.16,8.07)=3.36$ & $0.101^{*}$ \\
\hline IL-5 (pg/mL) & $0.16(0.16)$ & $0.97(0.59)$ & $1.22(0.51)$ & $(2,14)=1.40$ & 0.279 \\
\hline IL-6 (pg/mL) & $14.96(3.50)$ & $26.84(8.18)$ & $1.68(1.68)$ & $(2,14)=6.16$ & 0.012 \\
\hline IL-10 (pg/mL) & $0(0)$ & $0.58(0.58)$ & $0(0)$ & $(2,14)=1$ & 0.393 \\
\hline $\mathrm{IL}-12(\mathrm{p} 70)(\mathrm{pg} / \mathrm{mL})$ & $0.04(0.04)$ & $0.18(0.14)$ & $0(0)$ & $(1.12,7.9)=1.21$ & $0.312^{*}$ \\
\hline IL-13 (pg/mL) & $0(0)$ & $0.13(0.13)$ & $0(0)$ & $(2,14)=1$ & 0.393 \\
\hline IFN- $\gamma(\mathrm{pg} / \mathrm{mL})$ & $0.96(0.21)$ & $2.2(0.84)$ & $0.09(0.09)$ & $(1.1,7.7)=4.60$ & $0.063^{*}$ \\
\hline TNF- $\alpha(\mathrm{pg} / \mathrm{mL})$ & $0.98(0.19)$ & $1.75(0.57)$ & $0.11(0.11)$ & $(1.18,8.29)=6.47$ & $0.03^{*}$ \\
\hline sICAM-1 (ng/mL) & $0(0)$ & $4.64(4.64)$ & $16.58(10.87)$ & $(2,14)=2.10$ & 0.160 \\
\hline $\mathrm{CRP}(\mathrm{ng} / \mathrm{mL})$ & $0(0)$ & $10.25(10.25)$ & $5.89(5.89)$ & $(2,14)=0.54$ & 0.600 \\
\hline
\end{tabular}

Mediator levels are expressed as mean (SE).

* Greenhouse-Geisser correction for nonsphericity.

Furthermore, the authors showed that $\mathrm{BOP}$ was more robust in determining the degree of systemic inflammation than traditional classifications of periodontitis [29]. The current study, however, is novel in that it is the first to demonstrate a systemic sICAM-1 response to the accumulation of dental plaque.

The clinical results of the present study are consistent with previous experimental gingivitis studies $[16,18,19,23,25]$ and confirm that oral hygiene abstention results in dental plaque accumulation and gingival inflammation that can be reversed upon removal of the inflammatory stimulus (dental plaque) and reinstatement of oral hygiene procedures. Gingival inflammation at day 35 was significantly lower than reported at baseline. Optimal oral hygiene practice, in conjunction with the use of a powered toothbrush, during the resolution phase may explain the reduction in gingival inflammation to levels lower than baseline.

Five of the twelve mediators within the GCF demonstrated significant changes in levels over time; however, these changes were only demonstrated during the resolution phase and between day 35 and baseline, as opposed to during the induction phase. It is well documented in the literature that gingivitis is associated with increased GCF levels of inflammatory mediators [18-22]. The inability to detect such changes in the present study may have been due to the small sample size, the degree of gingival inflammation at baseline, and the high degree of individual variability in inflammatory responses. Nevertheless, the fact that the GCF levels of IL-2, IL- 6 , and TNF- $\alpha$ correlated with changes in plaque index scores, coincident with decreases in $\mathrm{BOP}$, during the resolution phase suggests that these mediators may reflect changes in bacterial challenge to the gingival tissues.

Cell adhesion molecules, such as sICAM-1, play an important role in the trafficking of leukocytes from the circulation into the tissues (reviewed in Cronstein and Weissmann, [30]). They are expressed on the cell surface of many cells including leukocytes and endothelial cells and can be shed from the cell surface by proteolytic cleavage [31]. Therefore, it is postulated that the elevated plasma levels of sICAM-1 detected at day 21, following the accumulation of dental plaque, may indicate leukocyte and/or endothelial activation [32]. In agreement with this, the studies carried out by Wahaidi and colleagues in 2009 [23] and 2011 [25] reported an increase in neutrophil hyperactivity following experimental gingivitis. It has been suggested that neutrophil activation/hyperactivity is an early response by circulating neutrophils to a bacterial challenge [33]. While levels of bacteremia were not measured in the present study, endotoxemia has been reported in experimental gingivitis and was associated with neutrophil activity in endotoxin-positive females [25]. Unexpectedly, plasma sICAM-1 levels did not return to baseline levels following resolution of gingivitis. While the half-life of sICAM-1 is currently unknown, the inability to detect significant changes in sICAM-1 levels following resolution of gingivitis may, in part, be due to the short duration of the resolution phase.

Despite the elevated plasma levels of sICAM-1 during the induction of gingivitis, these levels were not reflected within the oral fluids. This was unexpected, not only due to reports of a local contribution of ICAM-1 within the periodontal 
TABLE 3: Salivary mediator levels at baseline, peak of induction (day 21), and at resolution (day 35) of experimental gingivitis.

\begin{tabular}{|c|c|c|c|c|c|}
\hline & Baseline & Day 21 & Day 35 & $F$ statistic & $P$ value \\
\hline $\mathrm{IL}-1 \beta(\mathrm{pg} / \mathrm{mL})$ & $109.45(32.13)$ & $116.64(44.35)$ & 31.74 (11.79) & $(2,10)=2.95$ & 0.099 \\
\hline IL-2 (pg/mL) & $7.61(3.10)$ & $20.93(15.74)$ & $16.17(16.17)$ & $(1.04,5.19)=0.44$ & $0.543^{*}$ \\
\hline $\mathrm{IL}-4(\mathrm{pg} / \mathrm{mL})$ & $0.43(0.32)$ & $1.70(1.26)$ & $0.68(0.68)$ & $(1.12,5.62)=0.98$ & $0.375^{*}$ \\
\hline IL-5 (pg/mL) & $1.97(1.97)$ & $4.39(3.63)$ & $1.20(1.20)$ & $(2,10)=0.56$ & 0.587 \\
\hline IL-6 (pg/mL) & $103.77(43.00)$ & $120.10(45.46)$ & $73.06(31.41)$ & $(2,10)=0.95$ & 0.418 \\
\hline IL-10 (pg/mL) & $0(0)$ & $5.84(5.49)$ & $3.13(3.13)$ & $(1.00,5.01)=1.13$ & $0.337^{*}$ \\
\hline IL-12(p70) (pg/mL) & $0.24(0.24)$ & $1.81(1.55)$ & $0.94(0.94)$ & $(1.06,5.28)=0.96$ & $0.377^{*}$ \\
\hline IL-13 (pg/mL) & $0.10(0.10)$ & $3.79(3.52)$ & $2.89(2.89)$ & $(1.01,5.03)=1.04$ & $0.356^{*}$ \\
\hline IFN- $\gamma(\mathrm{pg} / \mathrm{mL})$ & $6.57(2.39)$ & $11.41(5.10)$ & $3.43(2.05)$ & $(2,10)=1.50$ & 0.269 \\
\hline TNF- $\alpha(\mathrm{pg} / \mathrm{mL})$ & $3.63(1.10)$ & $4.81(1.22)$ & $1.54(0.60)$ & $(2,10)=2.48$ & 0.133 \\
\hline sICAM-1 (ng/mL) & $20.46(7.90)$ & $8.63(5.65)$ & $4.97(4.97)$ & $(2,14)=2.46$ & 0.122 \\
\hline CRP (ng/mL) & $6.76(6.76)$ & $0(0)$ & $6.76(6.76)$ & $(2,14)=0.48$ & 0.127 \\
\hline
\end{tabular}

Mediator levels are expressed as mean (SE).

${ }^{*}$ Greenhouse-Geisser correction for nonsphericity.

TABle 4: Plasma mediator levels at baseline, peak of induction (day 21), and at resolution (day 35) of experimental gingivitis.

\begin{tabular}{|c|c|c|c|c|c|}
\hline & Baseline & Day 21 & Day 35 & $F$ statistic & $P$ value \\
\hline $\mathrm{IL}-1 \beta(\mathrm{pg} / \mathrm{mL})$ & $0(0)$ & $0(0)$ & $0(0)$ & - & - \\
\hline IL-2 (pg/mL) & $0(0)$ & $7.21(5.64)$ & $0(0)$ & $(2,14)=1.64$ & 0.229 \\
\hline $\mathrm{IL}-4(\mathrm{pg} / \mathrm{mL})$ & $0.15(0.15)$ & $0.28(0.28)$ & $0.42(0.31)$ & $(1.13,7.90)=0.44$ & $0.549^{*}$ \\
\hline IL-5 (pg/mL) & $40.44(14.67)$ & $26.16(8.43)$ & $44.39(19.44)$ & $(2,14)=1.50$ & 0.258 \\
\hline IL-6 (pg/mL) & $3.00(1.50)$ & $4.10(2.50)$ & $7.11(5.66)$ & $(1.11,7.75)=0.46$ & $0.539^{*}$ \\
\hline IL-10 (pg/mL) & $4.20(3.03)$ & $2.30(1.60)$ & $3.78(2.98)$ & $(2,14)=0.81$ & 0.466 \\
\hline IL-12(p70) (pg/mL) & $1.37(1.05)$ & $1.52(0.81)$ & $1.80(1.19)$ & $(2,14)=0.35$ & 0.712 \\
\hline IL-13 (pg/mL) & $0.55(0.55)$ & $0.12(0.12)$ & $0.55(0.55)$ & $(2,14)=1.00$ & 0.393 \\
\hline IFN- $\gamma(\mathrm{pg} / \mathrm{mL})$ & $0.44(0.24)$ & $0.44(0.33)$ & $0.77(0.77)$ & $(1.18,8.27)=0.24$ & $0.674^{*}$ \\
\hline TNF- $\alpha(\mathrm{pg} / \mathrm{mL})$ & $0(0)$ & $0(0)$ & $0(0)$ & - & - \\
\hline sICAM-1 (ng/mL) & $85.73(9.17)$ & $220.20(19.91)$ & $229.69(10.35)$ & $(2,14)=31.95$ & $<0.001$ \\
\hline $\mathrm{CRP}(\mathrm{ng} / \mathrm{mL})$ & $1456.75(652.23)$ & $873.95(355.55)$ & $1269.01(729.10)$ & $(1.13,7.94)=1.43$ & $0.273^{*}$ \\
\hline
\end{tabular}

Mediator levels are expressed as mean (SE).

* Greenhouse-Geisser correction for nonsphericity.

tissues but also because both of these fluids are serum derivatives. Gemmell et al. [34] demonstrated that ICAM-1 is constitutively expressed on periodontal endothelial cells in both gingival health and disease and that there is an increased expression of ICAM-1 on infiltrating cells in inflamed gingival tissues. Similarly, in experimental gingivitis, there is a gradient in ICAM-1 expression in the junctional epithelium with increased expression in areas in close proximity to dental plaque [35]. Further supporting this, soluble forms of ICAM-1 within the GCF have been associated with dental plaque accumulation and inflammation [36]. The reasons behind reduced levels of sICAM-1 in the oral fluids are unknown. While it may have been possible that technical issues associated with the elution or dilution of the GCF resulted in levels being under the detection limit of the assay, this is unlikely given the high levels within the plasma. On the other hand, the inability of circulating sICAM-1 molecules to transverse periodontal endothelial cells and/or periodontal tissues, the binding of sICAM-1 molecules to cells within the periodontal tissues and the degradation of sICAM-1 [37] seem more likely explanations. The discrepancy in levels of sICAM-1 between plasma and GCF is not limited to this study and has also been reported in smokers with periodontitis [37]. Similarly, the reduced salivary levels of sICAM-1 may reflect the inability of circulating sICAM-1 molecules to transverse either the endothelial cells or the acinar epithelial cells within the salivary glands, the degradation of sICAM-1 molecules, or the dilution of serum-derived sICAM-1 during saliva production. The mechanisms governing the regulation of sICAM-1 levels require further investigation.

In the present study, experimental gingivitis was not associated with systemic changes in levels of cytokines or CRP although individual variability in plasma CRP levels was noted. This is in agreement with other studies and has been proposed to be due to the mild nature of gingivitis [19] as well as the short duration of endotoxemia during the induction phase [25]. Indeed, in the study conducted by Wahaidi et al. [25] the earliest time point at which endotoxemia could be detected was in the last week of experimental gingivitis. As systemic levels of cytokines are influenced not only by 
the type of stimulant but also the duration of the stimulant, the authors speculated that extension of the induction phase would result in prolonged endotoxemia, which may have greater effects on systemic cytokine levels.

It is acknowledged that the sample size of the present pilot study is small; however, the results presented were significant and, thus, do reflect a real difference. It could also be argued that hormonal fluctuations, which were not monitored, may have influenced the results $[38,39]$. While this is a possibility, the fact that all subjects had a similar systemic response to dental plaque suggests that such a variation was not a major factor in the present study.

In conclusion, the results of the present study demonstrated that dental plaque accumulation resulted in an inflammatory response within the gingival tissues that was associated with a significant systemic sICAM-1 response. Within the limitations of this pilot study, one may conclude that the results support the concept that gingivitis adds to the systemic inflammatory burden of an individual; however, further studies are required to confirm these observations.

\section{Acknowledgments}

The authors gratefully acknowledge Dr. Rod Marshall, Dr. Hong Lien Do, Ms. Bonita Garton, and the study participants for their contributions to the clinical part of this study. This study was funded by the Australian Dental Research Foundation.

\section{References}

[1] A. Nanci and D. D. Bosshardt, "Structure of periodontal tissues in health and disease," Periodontology, vol. 40, no. 1, pp. 11-28, 2006.

[2] G. J. Seymour, P. J. Ford, M. P. Cullinan, S. Leishman, and K. Yamazaki, "Relationship between periodontal infections and systemic disease," Clinical Microbiology and Infection, vol. 13, no. 4, pp. 3-10, 2007.

[3] P. M. Ridker, C. H. Hennekens, J. E. Buring, and N. Rifai, "C-reactive protein and other markers of inflammation in the prediction of cardiovascular disease in women," The New England Journal of Medicine, vol. 342, no. 12, pp. 836-843, 2000.

[4] P. M. Ridker, N. Rifai, M. Pfeffer, F. Sacks, S. Lepage, and E. Braunwald, "Elevation of tumor necrosis factor- $\alpha$ and increased risk of recurrent coronary events after myocardial infarction," Circulation, vol. 101, no. 18, pp. 2149-2153, 2000.

[5] P. M. Ridker, N. Rifai, M. J. Stampfer, and C. H. Hennekens, "Plasma concentration of interleukin-6 and the risk of future myocardial infarction among apparently healthy men," Circulation, vol. 101, no. 15, pp. 1767-1772, 2000.

[6] S.-J. Hwang, C. M. Ballantyne, A. R. Sharrett et al., "Circulating adhesion molecules VCAM-1, ICAM-1, and E-selectin in carotid atherosclerosis and incident coronary heart disease cases: The Atherosclerosis Risk In Communities (ARIC) Study," Circulation, vol. 96, no. 12, pp. 4219-4225, 1997.

[7] P. M. Ridker, N. Rifai, L. Rose, J. E. Buring, and N. R. Cook, "Comparison of C-reactive protein and low-density lipoprotein cholesterol levels in the prediction of first cardiovascular events," The New England Journal of Medicine, vol. 347, no. 20, pp. 1557-1565, 2002.
[8] J. L. Ebersole, R. L. Machen, M. J. Steffen, and D. E. Willmann, "Systemic acute-phase reactants, C-reactive protein and haptoglobin, in adult periodontitis," Clinical and Experimental Immunology, vol. 107, no. 2, pp. 347-352, 1997.

[9] B. G. Loos, J. Craandijk, F. J. Hoek, P. M. E. Wertheim-van Dillen, and U. van der Velden, "Elevation of systemic markers related to cardiovascular diseases in the peripheral blood of periodontitis patients," Journal of Periodontology, vol. 71, no. 10, pp. 1528-1534, 2000.

[10] B. Noack, R. J. Genco, M. Trevisan, S. Grossi, J. J. Zambon, and E. De Nardin, "Periodontal infections contribute to elevated systemic C-reactive protien level," Journal of Periodontology, vol. 72, no. 9, pp. 1221-1227, 2001.

[11] S. Amar, N. Gokce, S. Morgan, M. Loukideli, T. E. Van Dyke, and J. A. Vita, "Periodontal disease is associated with brachial artery endothelial dysfunction and systemic inflammation," Arteriosclerosis, Thrombosis, and Vascular Biology, vol. 23, no. 7, pp. 1245-1249, 2003.

[12] L. Nibali, F. D’Aiuto, G. Griffiths, K. Patel, J. Suvan, and M. S. Tonetti, "Severe periodontitis is associated with systemic inflammation and a dysmetabolic status: a case-control study," Journal of Clinical Periodontology, vol. 34, no. 11, pp. 931-937, 2007.

[13] F. D’Aiuto, M. Parkar, L. Nibali, J. Suvan, J. Lessem, and M. S. Tonetti, "Periodontal infections cause changes in traditional and novel cardiovascular risk factors: results from a randomized controlled clinical trial," American Heart Journal, vol. 151, no. 5, pp. 977-984, 2006.

[14] M. S. Tonetti, F. D’Aiuto, L. Nibali et al., "Treatment of periodontitis and endothelial function," The New England Journal of Medicine, vol. 356, no. 9, pp. 911-920, 2007.

[15] B. A. Taylor, G. H. Tofler, H. M. R. Carey et al., "Full-mouth tooth extraction lowers systemic inflammatory and thrombotic markers of cardiovascular risk," Journal of Dental Research, vol. 85, no. 1, pp. 74-78, 2006.

[16] H. Löe, E. Theilade, and S. B. Jensen, "Experimental gingivitis in man," Journal of Periodontology, vol. 36, pp. 177-187, 1965.

[17] E. Gemmell, K. Yamazaki, and G. J. Seymour, "Destructive periodontitis lesions are determined by the nature of the lymphocytic response," Critical Reviews in Oral Biology and Medicine, vol. 13, no. 1, pp. 17-34, 2002.

[18] S. Offenbacher, S. Barros, L. Mendoza et al., "Changes in gingival crevicular fluid inflammatory mediator levels during the induction and resolution of experimental gingivitis in humans," Journal of Clinical Periodontology, vol. 37, no. 4, pp. 324-333, 2010.

[19] L. Trombelli, C. Scapoli, A. Carrieri, G. Giovannini, G. Calura, and R. Farina, "Interleukin-1 $\beta$ levels in gingival crevicular fluid and serum under naturally occurring and experimentally induced gingivitis," Journal of Clinical Periodontology, vol. 37, no. 8, pp. 697-704, 2010.

[20] O. M. Andriankaja, S. P. Barros, K. Moss et al., "Levels of serum interleukin (IL)- 6 and gingival crevicular fluid of IL- $1 \beta$ and prostaglandin E2 among non-smoking subjects with gingivitis and type 2 diabetes," Journal of Periodontology, vol. 80, no. 2, pp. 307-316, 2009.

[21] S. Offenbacher, S. P. Barros, R. E. Singer, K. Moss, R. C. Williams, and J. D. Beck, "Periodontal disease at the biofilmgingival interface," Journal of Periodontology, vol. 78, no. 10, pp. 1911-1925, 2007.

[22] R. Deinzer, U. Weik, V. Kolb-Bachofen, and A. Herforth, "Comparison of experimental gingivitis with persistent gingivitis: 
differences in clinical parameters and cytokine concentrations," Journal of Periodontal Research, vol. 42, no. 4, pp. 318-324, 2007.

[23] V. Y. Wahaidi, S. A. Dowsett, G. J. Eckert, and M. J. Kowolik, "Neutrophil response to dental plaque by gender and race," Journal of Dental Research, vol. 88, no. 8, pp. 709-714, 2009.

[24] H. J. Wright, I. L. C. Chapple, and J. B. Matthews, "Levels of TGF $\beta 1$ in gingival crevicular fluid during a 21-day experimental model of gingivitis," Oral Diseases, vol. 9, no. 2, pp. 88-94, 2003.

[25] V. Y. Wahaidi, M. J. Kowolik, G. J. Eckert, and D. M. Galli, "Endotoxemia and the host systemic response during experimental gingivitis," Journal of Clinical Periodontology, vol. 38, no. 5, pp. 412-417, 2011.

[26] M. J. Kowolik, S. A. Dowsett, J. Rodriguez, M. De La Rosa, and G. J. Eckert, "Systemic neutrophil response resulting from dental plaque accumulation," Journal of Periodontology, vol. 72, no. 2, pp. 146-151, 2001.

[27] J. Silness and H. Löe, "Periodontal disease in pregnancy. Correlation between oral hygiene and periodontal condition," Acta Odontologica Scandinavica, vol. 22, pp. 121-135, 1964.

[28] J. M. Bland and D. G. Altman, "Analysis of continuous data from small samples," British Medical Journal, vol. 338, article a3166, 2009.

[29] J. D. Beck and S. Offenbacher, "Relationships among clinical measures of periodontal disease and their associations with systemic markers," Annals of Periodontology, vol. 7, no. 1, pp. 7989, 2002.

[30] B. N. Cronstein and G. Weissmann, "The adhesion molecules of inflammation," Arthritis and Rheumatism, vol. 36, no. 2, pp. 147-157, 1993.

[31] J. F. M. Leeuwenberg, E. F. Smeets, J. J. Neefjes et al., "E-selectin and intercellular adhesion molecule-1 are released by activated human endothelial cells in vitro," Immunology, vol. 77, no. 4, pp. 543-549, 1992.

[32] C. Maple, G. Kirk, M. Mclaren, D. Veale, and J. J. F. Belch, "A circadian variation exists for soluble levels of intercellular adhesion molecule-1 and E-selectin in healthy volunteers," Clinical Science, vol. 94, no. 5, pp. 537-540, 1998.

[33] H. R. Hill, W. J. Warwick, J. Dettloff, and P. G. Quie, "Neutrophil granulocyte function in patients with pulmonary infection," Journal of Pediatrics, vol. 84, no. 1, pp. 55-58, 1974.

[34] E. Gemmell, L. J. Walsh, N. W. Savage, and G. J. Seymour, "Adhesion molecule expression in chronic inflammatory periodontal disease tissue," Journal of Periodontal Research, vol. 29, no. 1, pp. 46-53, 1994.

[35] N. A. Moughal, E. Adonogianaki, M. H. Thornhill, and D. F. Kinane, "Endothelial cell leukocyte adhesion molecule-1 (ELAM-1) and intercellular adhesion molecule-1 (ICAM-1) expression in gingival tissue during health and experimentallyinduced gingivitis," Journal of Periodontal Research, vol. 27, no. 6, pp. 623-630, 1992.

[36] N. Molé, A. Kennel-de March, G. Martin, N. Miller, M. C. Béné, and G. C. Faure, "High levels of soluble Intercellular Adhesion Molecule-1 (ICAM-1) in crevicular fluid of periodontitis patients with plaque," Journal of Clinical Periodontology, vol. 25, no. 9, pp. 754-758, 1998.

[37] H. S. Fraser, R. M. Palmer, R. F. Wilson, P. Y. Coward, and D. A. Scott, "Elevated systemic concentrations of soluble ICAM1 (sICAM) are not reflected in the gingival crevicular fluid of smokers with periodontitis," Journal of Dental Research, vol. 80, no. 7, pp. 1643-1647, 2001.
[38] P. Holm-Pedersen and H. Löe, "Flow of gingival exudate as related to menstruation and pregnancy," Journal of Periodontal Research, vol. 2, no. 1, pp. 13-20, 1967.

[39] U. Baser, A. Cekici, S. Tanrikulu-Kucuk, A. Kantarci, E. Ademoglu, and F. Yalcin, "Gingival inflammation and interleukin $1-\beta$ and tumor necrosis factor-alpha levels in gingival crevicular fluid during the menstrual cycle," Journal of Periodontology, vol. 80, no. 12, pp. 1983-1990, 2009. 


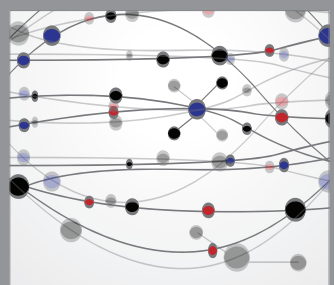

The Scientific World Journal
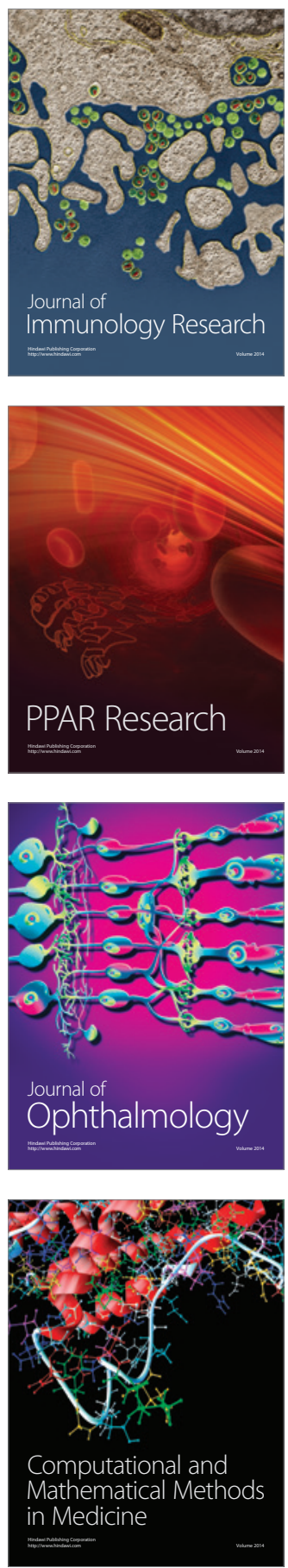

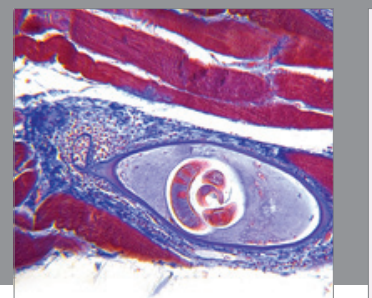

Gastroenterology

Research and Practice
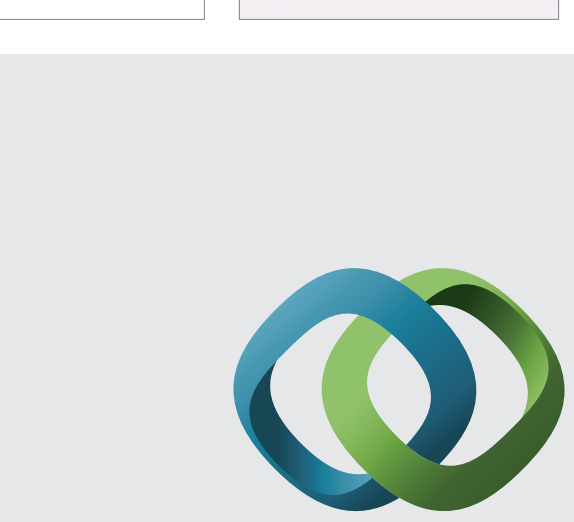

\section{Hindawi}

Submit your manuscripts at

http://www.hindawi.com
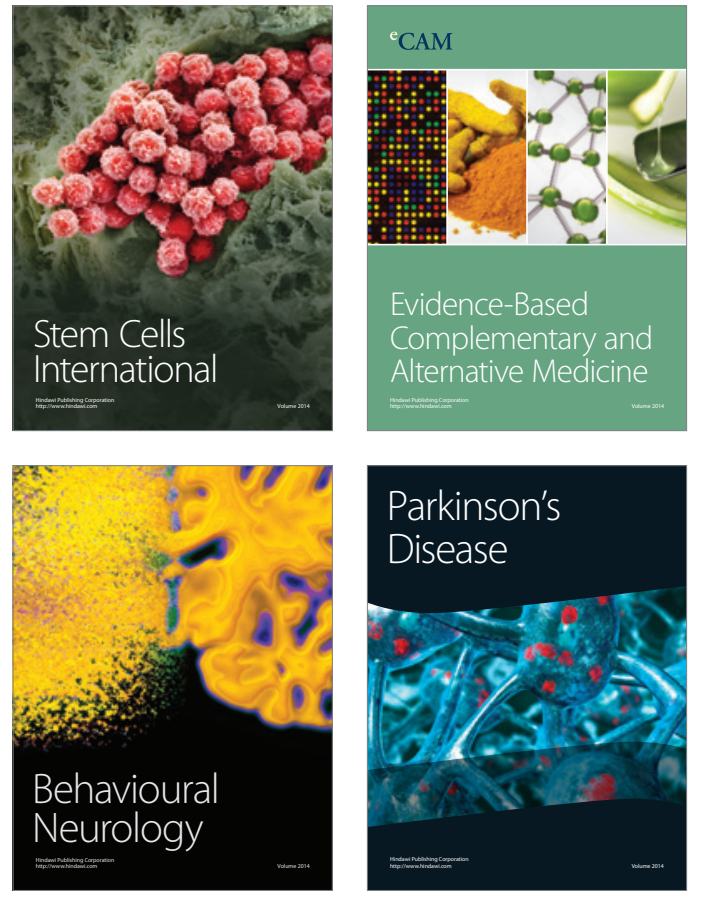
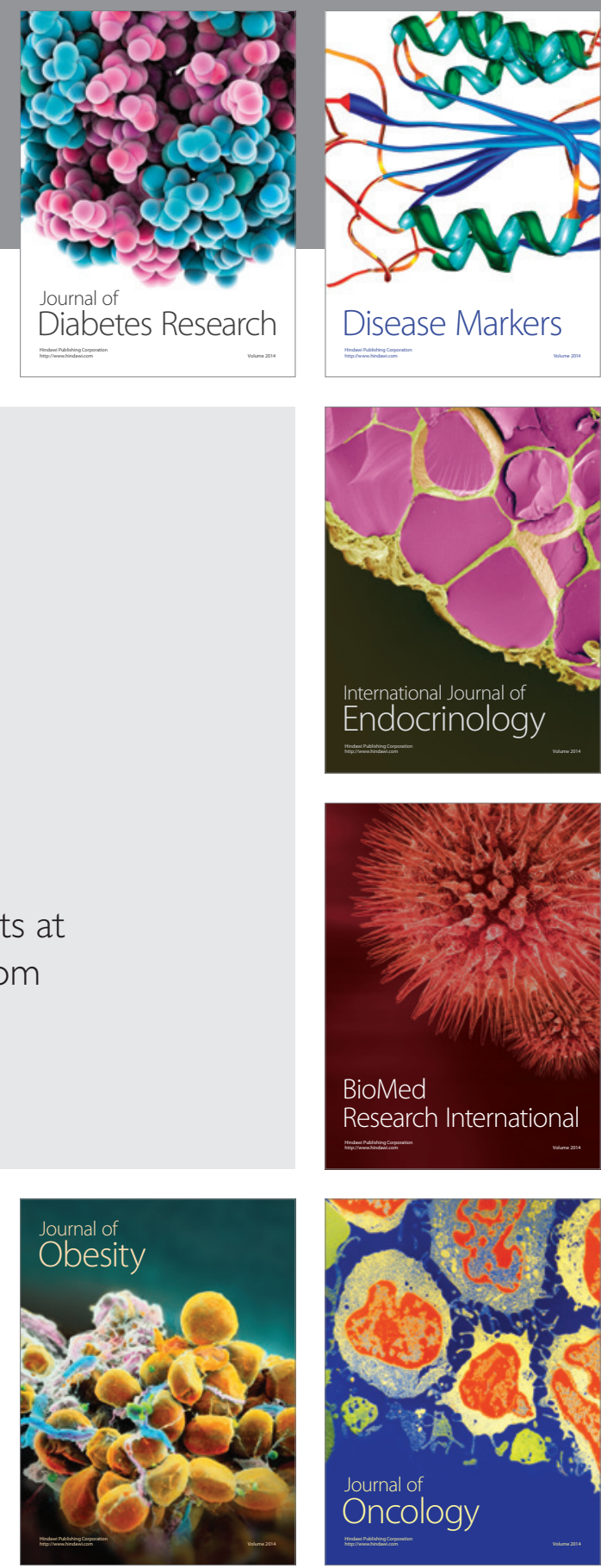

Disease Markers
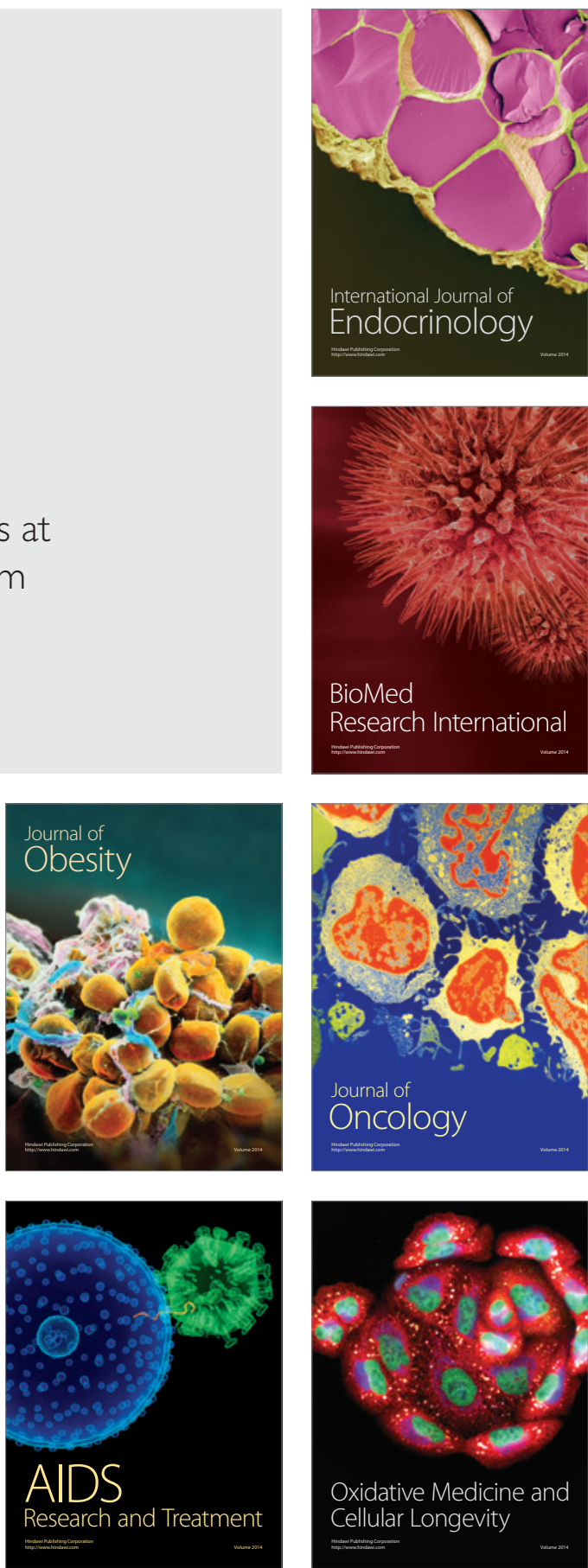